\title{
Particle-in-Cell Simulations of Synchrotron Maser Emission and Associated Particle Acceleration in Relativistic Shocks
}

\author{
M. Iwamoto, ${ }^{a, *}$ T. Amano, ${ }^{b}$ Y. Matsumoto, ${ }^{c}$ S. Matsukiyo ${ }^{a}$ and M. Hoshino ${ }^{b}$ \\ ${ }^{a}$ Faculty of Engineering Sciences, Kyushu University, \\ 6-1, Kasuga-koen, Kasuga, Fukuoka, 816-8580, Japan \\ ${ }^{b}$ Department of Earth and Planetary Science, University of Tokyo, \\ 7-3-1 Hongo, Bunkyo-ku, Tokyo 113-0033, Japan \\ ${ }^{c}$ Department of Physics, Chiba University, \\ 1-33 Yayoi, Inage-ku, Chiba, Chiba 263-8522, Japan \\ E-mail: iwamoto@esst.kyushu-u.ac.jp
}

We study synchrotron maser emission and associated particle acceleration at electron-ion relativistic shocks by PIC simulations. Relativistic shocks emit intense electromagnetic waves from the shock front via the synchrotron maser instability. This synchrotron maser emission induces electrostatic plasma waves (i.e., wakefield) and transverse filamentary structures in the upstream region via the stimulated/induced Raman scattering and filamentation instability, respectively. Some lucky particles in the phase of the deceleration by the wakefield enter the filamentary structures. Then they are reflected by the wakefield and decoupled from the upstream bulk flow. These decoupled particles can feel the motional electric field and efficiently accelerated.

\footnotetext{
*** 37th International Cosmic Ray Conference (ICRC2021), ***

*** 12-23 July 2021 ***

*** Berlin, Germany - Online ***
}

\footnotetext{
${ }^{*}$ Presenter
} 


\section{Introduction}

Relativistic shocks are ubiquitous in the Universe as a consequence of interaction between relativistic plasma outflow and interstellar medium and believed to be an efficient particle accelerators. Although particle acceleration at relativistic shocks is often invoked for the generation mechanism of the ultra-high-energy cosmic rays (UHECRs), which is a long-standing in astrophysics, the detailed acceleration mechanism remains unsolved yet.

It is well-known that relativistic shocks emit coherent electromagnetic waves [1-8], which are assumed to originate from the synchrotron maser instability (SMI) [9]. 1D particle-in-cell (PIC) simulations of relativistic shocks demonstrated that longitudinal electrostatic waves, which are called wakefields, are induced in the wake of the large- amplitude electromagnetic waves via the stimulated Raman scattering (SRS) and that nonthermal particles are generated during the nonlinear collapse of the wakefields $[10,11]$. This particle acceleration mechanism is similar to the wakefield acceleration (WFA) which has been widely studied in laboratory plasma [12] and may explain the origin of UHECRs [13]. Recently, our high-resolution 2D PIC simulation [14] confirmed that the particle acceleration associated with the wakefield works even in realistic multidimensional shocks. In this study, we investigate the acceleration mechanism in more detail and show that particles are pre-accelerated by the wakefield and then enter the phase of the main acceleration by the motional electric field operates.

\section{Simulation Setting}

We employ a fully-kinetic 2D PIC code $[15,16]$ which suppresses the unphysical electromagnetic wave radiation by the numerical Cherenkov instability [17]. Our simulation configuration is illustrated in Figure 1. The number of grids is $N_{x} \times N_{y}=16000 \times 1600$. The cold ion-electron plasma flow with the ion-to-electron mass ratio $m_{i} / m_{e}=50$ is traveling in the $-x$ direction with the bulk Lorentz factor $\gamma_{1}=40$. The incoming particles are reflected by the right-hand cinducting wall and induce the shock propagating in the $+x$ direction. The particle number per cell is $N_{1} \Delta x^{2}=64$ for both electrons and ions and the grid size is $\Delta x /\left(c / \omega_{p e}\right)=1 / 40$, where $\omega_{p e}=\sqrt{4 \pi N_{1} e / \gamma_{1} m_{e}}$ is the proper electron plasma frequency. The time step is $\omega_{p e} \Delta t=1 / 40$. The ambient magnetic field $B_{1}$ is in the $z$ direction and the ion and electron magnetization parameters are $\sigma_{i}=B_{1}^{2} / 4 \pi N_{1} m_{e} c^{2}=0.1$ and $\sigma_{e}=\left(m_{i} / m_{e}\right) \sigma_{i}=5$.

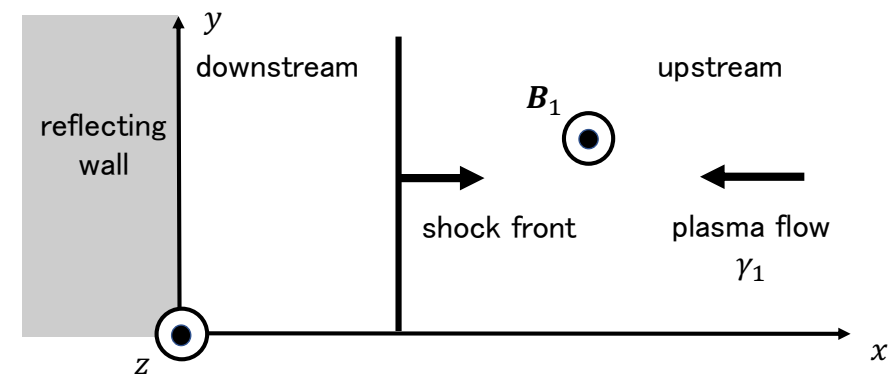

Figure 1: Schematic picture of simulation configuration. 


\section{Shock Structure}

Figure 2 shows the shock structure at $\omega_{p e} t=2000$. From top to bottom, the electron number density $N_{e}$, the ion number density $N_{i}$, the out-of-plane magnetic field $B_{z}$, the 1D profile for $B_{z}$ along $y /\left(c / \omega_{p e}\right)=20$, the longitudinal electric field $E_{x}$, the transversely averaged electrostatic field $\left\langle E_{x}\right\rangle$, and the electron and ion phase space densities $x-u_{x s}$ integrated over the $y$ direction are shown. The large-amplitude electromagnetic waves are clearly seen in the upstream. The precursor wave induces a large-scale longitudinal electric field by the SRS, which is so-called wakefield as already discussed by [11, 14]. The ion and electron density filaments are generated in the upstream. We think the filaments are attributed to the filamentation instability (FI) due to the intense precursor waves [18-21]. The wakefield breaks up in the region $1150 \lesssim x /\left(c / \omega_{p e}\right) \lesssim 1350$, indicating that the SRS enters the nonlinear phase. Both electrons and ions are strongly heated and accelerated there.

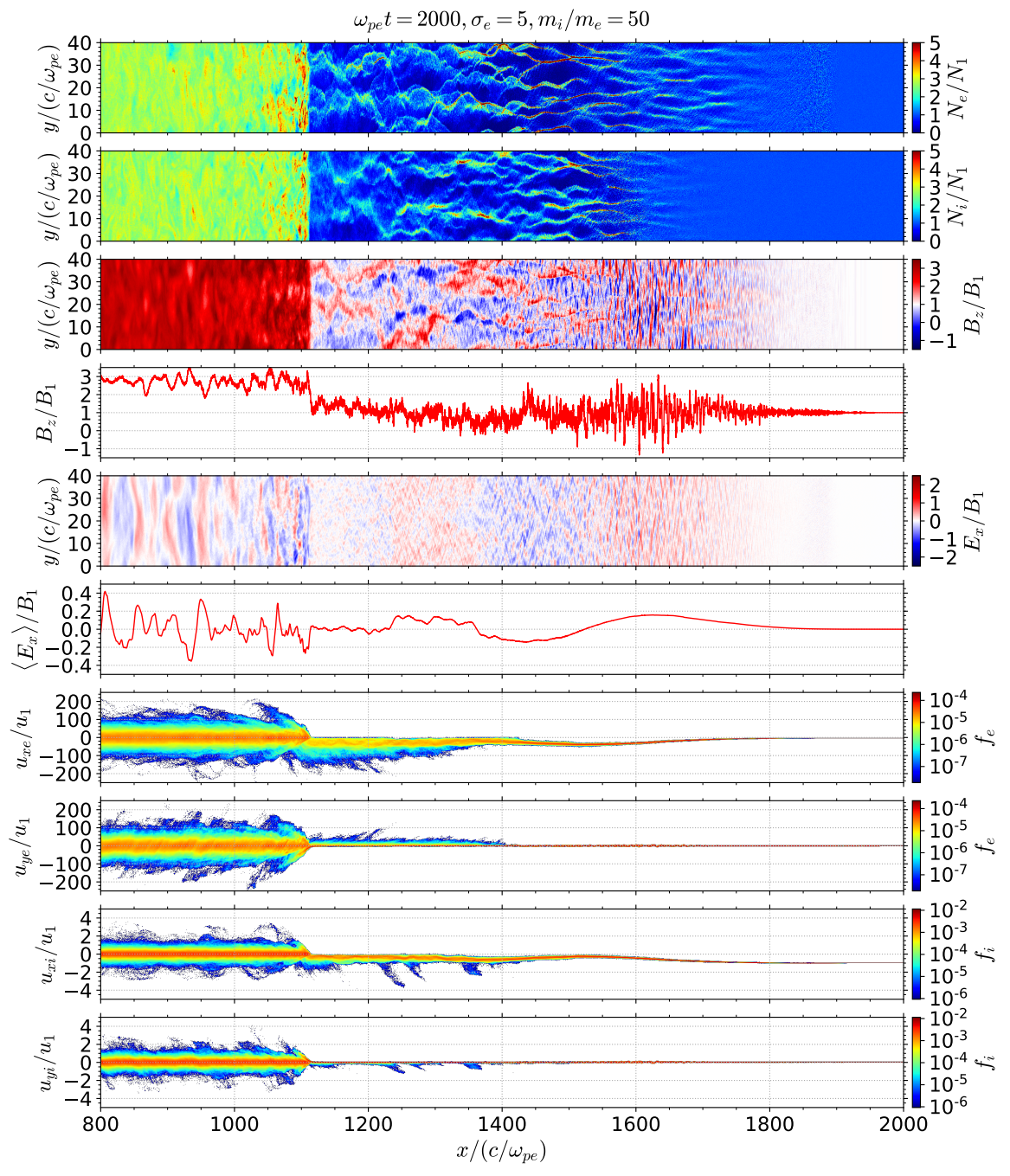

Figure 2: Global shock structure at $\omega_{p e} t=2000$. 
Figure 3 shows the upstream particle energy spectra measured in the simulation frame (left) and plasma rest frame (right). The electron and ion spectra are shown in red and blue, respectively. The electron Lorentz factor is scaled by the mass ratio $\gamma_{e} m_{e} / m_{i}$. Energy equipartition is roughly achieved due to the electron-ion coupling via the wakefield [10,11, 14]. The clear nonthermal tails are shown in the left panel of Figure 3 for both electrons and ions.
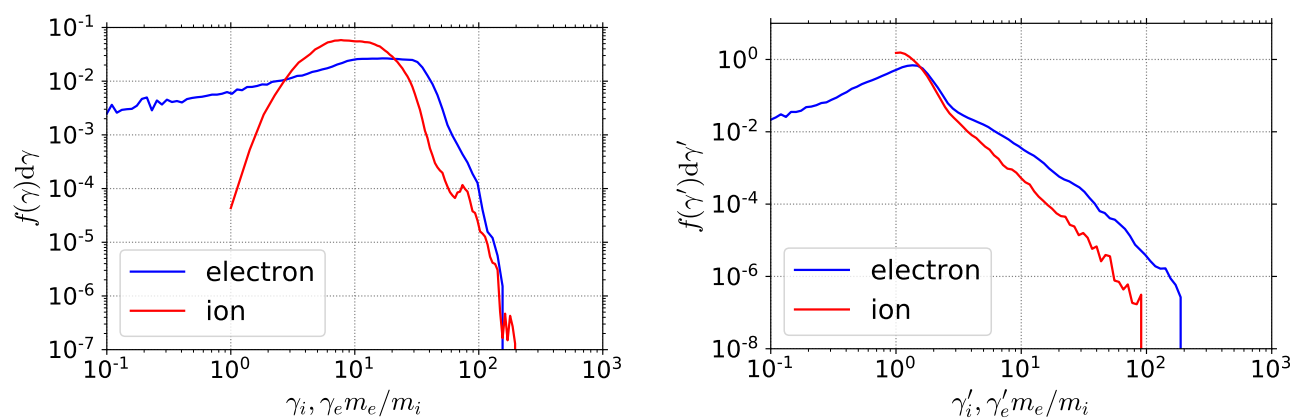

Figure 3: Upstream particle energy spectra measured in the simulation frame (left) and plasma rest frame (right).

\section{Acceleration Mechanism}

The left panels of Figure 4 show typical trajectory of a nonthermal electron in the $x-y$ (top) and $x-\gamma$ plane (bottom). The energetic electron initially oscillate in the $x$ direction due to the laminar wakefield. It begins to travel along the $+y$ direction and seems to gain energy from the motional electric field $E_{y} \sim-B_{1}$. This particle acceleration continues until the electron enters the shock at $x /\left(c / \omega_{p e}\right) \sim 1250$. We think this acceleration by the motional electric field can be explained as follows.
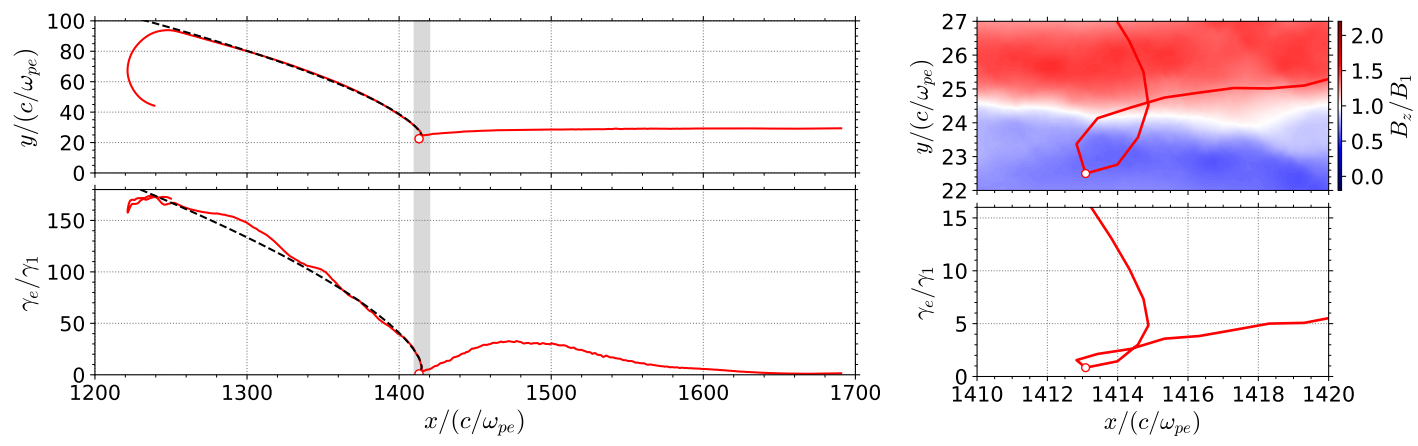

Figure 4: Left: Nonthermal electron trajectory in the $x-y$ (top) and $x-\gamma$ (bottom) space. The black dashed lines are theoretical estimate. Right: Enlarged view of the gray-shaded region. The map of $B_{z}$ corresponds to the time $\omega_{p e} t=2080$ when the electrons are reflected by the wakefield. 
The basic equations are relativistic equations of motion:

$$
\begin{aligned}
& m_{s} c \frac{\mathrm{d}}{\mathrm{d} t} \gamma_{s} \beta_{x s}=q_{s} \beta_{y s} B_{1}, \\
& m_{s} c \frac{\mathrm{d}}{\mathrm{d} t} \gamma_{s} \beta_{y s}=-q_{s}\left(\beta_{1}+\beta_{x s}\right) B_{1}
\end{aligned}
$$

Here we neglect the electromagnetic waves, wakefields, and filaments and consider only the 0-th order electromagnetic fields. The subscript $s=i, e$ indicates the particle species. For $\gamma_{1} \gg 1$, which is satidfied in our simulations, these equations reduce to

$$
\begin{aligned}
& x_{s} \simeq x_{0 s}-c \beta_{1} t \\
& y_{s} \simeq y_{0 s} \pm \gamma_{0 s} \gamma_{1}\left(\beta_{1}+\beta_{0 s}\right) \frac{c}{\omega_{c s}}\left(1-\cos \frac{\omega_{c s} t}{\gamma_{0 s} \gamma_{1}^{2}\left(1+\beta_{0 s} \beta_{1}\right)}\right) \\
& \gamma_{s} \simeq \gamma_{0 s} \gamma_{1}^{2}\left[\left(1+\beta_{0 s} \beta_{1}\right)-\beta_{1}\left(\beta_{1}+\beta_{0 s}\right) \cos \frac{\omega_{c s} t}{\gamma_{0 s} \gamma_{1}^{2}\left(1+\beta_{0 s} \beta_{1}\right)}\right]
\end{aligned}
$$

where $\omega_{c s}=e B_{1} / \gamma_{1} m_{s} c^{2}>0$ is the relativistic cyclotron frequency and the subscript 0 indicates the initial quantities at the time when particles are picked up by the bulk flow. The positive (negative) sign in $y_{s}$ corresponds to electrons (ions). Here we assume $\boldsymbol{\beta}_{0 s}=+\beta_{0 s} \hat{\boldsymbol{x}}$. We determined the initial quantities: $x_{0}, y_{0}$, and $\beta_{0}$ from the simulation result and show the analytical solutions in Figure 4 by black dashed lines. The theoretical trajectory gives a good agreement with both ion and electron, and clearly indicates that the particles are accelerated by the motional electric field. According to Equation 5, the maximum Lorentz factor is estimated as

$$
\gamma_{\max , s} \sim \gamma_{0 s} \gamma_{1}^{2}
$$

Here we have neglected the factors on the order of unity.

The above estimate assumes that the nonthermal particle travel in the opposite direction of the bulk flow just before accelerate by the motional electric field. The trajectories in the left panels of Figure 4 indeed demonstrate that nonthermal particles propagate toward the $+x$ direction. Such particle are decoupled from the bulk flow and feel the motional electric field [22]. As can be seen in the left panels of Figure 4, the nonthermal particle enter the weekly magnetized region due to the filaments, indicating that the filaments inject the particles into the acceleration process.

The time evolution of the Lorentz factor is shown in Figure 5. Here $\Delta \gamma_{x}$ (green) and $\Delta \gamma_{x}$ (blue) are the work done by the electric field $E_{x}$ and $E_{y}$, respectively and calculated as follows:

$$
\begin{aligned}
\Delta \gamma_{x} & =-\frac{e}{m_{e} c^{2}} \int_{t_{0}}^{t} E_{x} v_{x} \mathrm{~d} t, \\
\Delta \gamma_{y} & =-\frac{e}{m_{e} c^{2}} \int_{t_{0}}^{t} E_{y} v_{y} \mathrm{~d} t,
\end{aligned}
$$

where $\omega_{p e} t_{0}=2080$ corresponds to the vertical gray dashed line. The nonthermal particles are first accelerated by the wakfield and then decoupled from the bulk flow. This pre-acceleration may be explained as follows. The incoming electrons are decelerated by the wakefield and begin to gyrate. Some lucky electrons can enter the filaments, where the electrostatic field $-e E_{x}$ is larger than the 
Lorentz force $-e \beta_{y} B_{z}$ because the $B_{z}$ almost vanishes inside the filaments (see the left panels of Figure 4). They are then trapped by the wakefield and accelerated just like the WFA in laboratory plasmas until $-e \beta_{y} B_{z}$ exceeds $-e E_{x}$. After detrapped by the wakefield, they are further accelerated by the motional electric field during convecting toward the downstream.

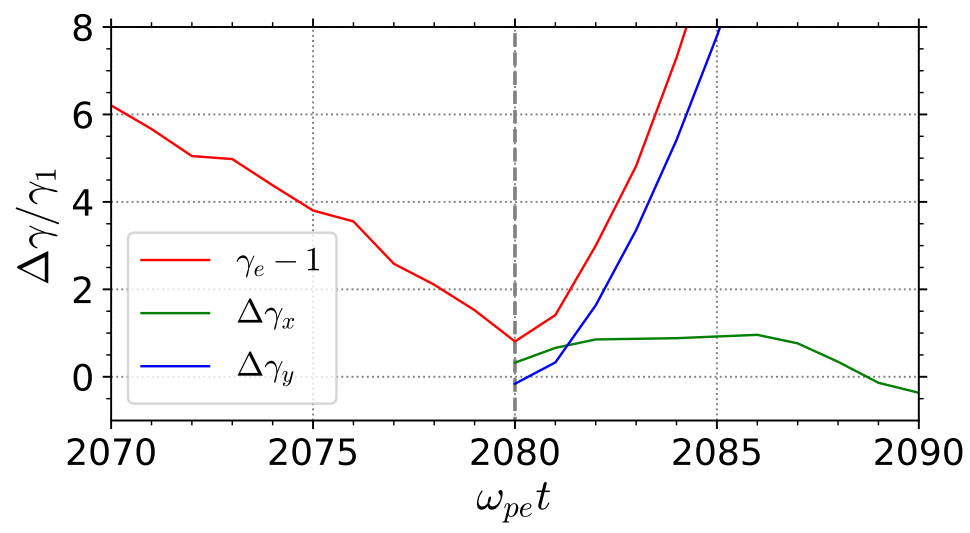

Figure 5: Time evolution of the Lorentz factor.

Now we estimate $\gamma_{0 e}$. If the pre-acceleration is described by the WFA, $\gamma_{0 e}$ satisfies

$$
\gamma_{0 e}=1+\Delta \gamma_{x}
$$

By definition, $\Delta \gamma_{x}$ is written as

$$
\frac{\Delta \gamma_{x}}{\gamma_{1}}=\frac{e E_{w a k e} L}{\gamma_{1} m_{e} c^{2}}=\sqrt{\sigma_{e}} \frac{E_{\text {wake }}}{B_{1}} \frac{L}{c / \omega_{p e}}
$$

where $E_{\text {wake }}$ and $L$ are the wakefield amplitude and acceleration length, respectively. $E_{\text {wake }}$ can be evaluated as [11]

$$
\frac{E_{w a k e}}{B_{1}}=\frac{1}{\gamma_{1} \sqrt{\sigma_{e}}} \frac{\eta a^{2}}{\sqrt{1+\eta a^{2}}},
$$

where $a=e \delta E / m_{e} c \omega$ is the wave strength parameter and $\eta$ represents the wave polarization: $\eta=1$ for circular polarization and $\eta=1 / 2$ for linear polarization. The wave strength parameter $a$ can be estimated as

$$
a \sim \gamma_{1} \gg 1
$$

for $\sigma_{i}=0.1[4,6]$. Our simulation results indicate

$$
\frac{L}{c / \omega_{p e}} \sim 1
$$

Substituting 11, 12 and 13 into 10, we have

$$
\frac{\Delta \gamma_{x}}{\gamma_{1}} \sim 1
$$


Here we have neglected the factors on the order of unity. This estimate gives a good agreement with our simulation results (see the green line in Figure 5). We finally obtain

$$
\gamma_{0 e} \sim \gamma_{1}
$$

$\gamma_{0 i}$ can be estimated in the same manner and we have

$$
\gamma_{0 i} \sim 1+\frac{m_{e}}{m_{i}} \gamma_{1}
$$

Substituting 15 and 16 into 5, the maximum Lorentz factor can be estimated as

$$
\begin{aligned}
\gamma_{\max , e} & \sim \gamma_{1}^{3}, \\
\gamma_{\max , i} & \sim\left(1+\frac{m_{e}}{m_{i}} \gamma_{1}\right) \gamma_{1}^{2} .
\end{aligned}
$$

The above estimate indicates that highly-relativistic shocks $\gamma_{1} \gg 1$ can generate energetic particles.

\section{Summary}

In this work, we investigated the particle acceleration associated with the synchrotron maser emission in relativistic ion-electron shocks by 2D PIC simulations. We found that particles are pre-accelerated by the wakefield within the filaments and then further accelerated by the motional electric field. The maximum Lorentz factors of the electron and ion can be estimated as $\gamma_{\text {max }, e} \sim \gamma_{1}^{3}$ and $\gamma_{\max , i} \sim\left(1+m_{e} \gamma_{1} / m_{i}\right) \gamma_{1}^{2}$, respectively.

\section{References}

[1] A.B. Langdon, J. Arons and C.E. Max, Magnetosonic shocks in electron-positron plasmas, PRL 61 (1988) 779.

[2] Y.A. Gallant, M. Hoshino, A.B. Langdon, J. Arons and C.E. Max, Relativistic, perpendicular shocks in electron-positron plasmas, ApJ 391 (1992) 73.

[3] M. Hoshino, J. Arons, Y.A. Gallant and A.B. Langdon, Relativistic magnetosonic shock waves in synchrotron sources - shock structure and nonthermal acceleration of positrons, ApJ 390 (1992) 454.

[4] M. Iwamoto, T. Amano, M. Hoshino and Y. Matsumoto, Persistence of precursor waves in two-dimensional relativistic shocks, ApJ 840 (2017) 52.

[5] M. Iwamoto, T. Amano, M. Hoshino and Y. Matsumoto, Precursor wave emission enhanced by weibel instability in relativistic shocks, ApJ 858 (2018) 93.

[6] I. Plotnikov, A. Grassi and M. Grech, Perpendicular relativistic shocks in magnetized pair plasma, MNRAS 477 (2018) 5238.

[7] I. Plotnikov and L. Sironi, The synchrotron maser emission from relativistic shocks in fast radio bursts: 1d pic simulations of cold pair plasmas, MNRAS 485 (2019) 3816. 
[8] A.-N. Babul and L. Sironi, The synchrotron maser emission from relativistic magnetized shocks: dependence on the pre-shock temperature, MNRAS 499 (2020) 2884 [2006.03081].

[9] M. Hoshino and J. Arons, Preferential positron heating and acceleration by synchrotron maser instabilities in relativistic positron-electron-proton plasmas, PhFlB 3 (1991) 818.

[10] Y. Lyubarsky, Electron-ion coupling upstream of relativistic collisionless shocks, ApJ 652 (2006) 1297.

[11] M. Hoshino, Wakefield acceleration by radiation pressure in relativistic shock waves, ApJ 672 (2008) 940.

[12] T. Tajima and J.M. Dawson, Laser electron accelerator, PRL 43 (1979) 267.

[13] P. Chen, T. Tajima and Y. Takahashi, Plasma wakefield acceleration for ultrahigh-energy cosmic rays, PRL 89 (2002) 161101.

[14] M. Iwamoto, T. Amano, M. Hoshino, Y. Matsumoto, J. Niemiec, A. Ligorini et al., Precursor Wave Amplification by Ion-Electron Coupling through Wakefield in Relativistic Shocks, ApJL 883 (2019) L35.

[15] Y. Matsumoto, T. Amano and M. Hoshino, Electron acceleration in a nonrelativistic shock with very high alfvén mach number, PRL 111 (2013) 215003.

[16] Y. Matsumoto, T. Amano, T.N. Kato and M. Hoshino, Stochastic electron acceleration during spontaneous turbulent reconnection in a strong shock wave, Science 347 (2015) 974.

[17] N. Ikeya and Y. Matsumoto, Stability property of numerical cherenkov radiation and its application to relativistic shock simulations, PASJ 67 (2015) 64.

[18] P.K. Kaw, G. Schmid and T. Wilcox, Filamentation and trapping of electromagnetic radiation in plasmas, PhFl 16 (1973) 1522.

[19] J.F. Drake, P.K. Kaw, Y.C. Lee, G. Schmid, C.S. Liu and M.N. Rosenbluth, Parametric instabilities of electromagnetic waves in plasmas, PhFl 14 (1974) 778.

[20] C.E. Max, J. Arons and A.B. Langdon, Self-Modulation and Self-Focusing of Electromagnetic Waves in Plasmas, PRL 33 (1974) 209.

[21] E. Sobacchi, Y. Lyubarsky, A.M. Beloborodov and L. Sironi, Self-modulation of fast radio bursts, MNRAS 500 (2020) 272 [2010.08282].

[22] L. Sironi, A. Spitkovsky and J. Arons, The maximum energy of accelerated particles in relativistic collisionless shocks, ApJ 771 (2013) 54. 